\title{
A Stochastic Optimal Control Method of Home Energy Based on Adaptive Dynamic Programming
}

\author{
Yi Liü, 2, a, Yun Zhang ${ }^{1, ~ b, ~}{ }^{\text {* }}$, Sizhe Chen ${ }^{1, c}$ \\ ${ }^{1}$ School of Automation, Guangdong University of Technology, Guangzhou 510006, China \\ ${ }^{2}$ School of Information Science and Technology, Zhongkai University of Agriculture and \\ Engineering, Guangzhou 510225, China \\ aemail: baddielao@126.com, bemail: yun@gdut.edu.cn, cemail: cszscut@126.com
}

Keywords: Adaptive Dynamic Programming; Stochastic Control; Home Energy Optimization; Time-varying Parameter; Smart Grid

\begin{abstract}
Home energy optimization (HEO) is an infinite horizon optimization problem, only adaptive dynamic programming (ADP) can solve infinite horizon optimization problem. But due to the existence of time-varying parameters, ADP can not converge to the optimal and can not be applied directly to the optimal control of home energy. According to the periodic characteristics of the time-varying parameters, a stochastic optimal control method is proposed in the paper based on ADP. The influence of time-varying parameters in the iteration of ADP is eliminated by the proposed method. Simulation results show the performance of the proposed method.
\end{abstract}

\section{Introduction}

Household energy management is an important part in smart grid. HEO is a hot research topic in the literature[1-7]. There are dynamic appliances such as battery within the system of HEO. Furthermore, all the costs of the time steps in the future should be considered. Therefore, HEO is an infinite horizon dynamic optimization problem. Dynamic programming (DP) can solve dynamic optimization accurately, but it will confront the "curse of dimensionality" [8] while the number of time step increases. The intelligent optimization algorithms such as particle swarm optimization are also applied to solve the HEO problem in the literature. But it is hard to get the optimal solution while the number of time step increases. Therefore, the infinite horizon problem is usually simplified into finite horizon problem. The horizon is usually set as one day, and the number of time step is 24 as the time interval is usually set as one hour.

ADP could solve the "curse of dimensionality" and infinite horizon optimization problem [9][10]. In 2011, Werbos, Venayamoorthy, and Powell proposed the framework of ADP based energy optimization for smart grid [11-13].

The convergence and optimality of ADP without time-varying parameter are proven [10][14][15]. But ADP can not be applied directly to HEO as there are time-varying parameters within HEO.

Dynamic price and power consumptions of uncontrollable appliance are the most common time-varying parameters in HEO. Dynamic price can be considered as a periodic function as the daily dynamic price is similar. On the other hand, though the power consumption of every uncontrollable appliance is determined by user, the daily probability density function (PDF) of power consumption of every uncontrollable appliance is similar as the daily user behavior is similar. In this paper, the time-varying parameters are eliminated by the periodic feature, and a new ADP algorithm is proposed to solve HEO with time-varying parameters.

\section{ADP Algorithm}

Let discrete-time system be

$$
x_{k+1}=F\left(x_{k}, u_{k}\right)
$$

where $x_{k} \in \mathbb{R}^{n}$ is state vector, $u_{k} \in \mathbb{R}^{m}$ is control vector, and $k$ is the index of time step, 
$k=0,1,2, \cdots . \forall x_{k}, u_{k}, F\left(x_{k}, u_{k}\right)$ is continuous. (1) is controllable and feedback control of state is applied. That is $u_{k}=u\left(x_{k}\right)$. The goal of optimization is to find the optimal control law $u^{*}\left(x_{k}\right)$ and minimize the performance index function as follow

$$
J\left(x_{k}\right)=\sum_{t=k}^{\infty} \gamma^{t-k} U\left(x_{t}, u\left(x_{t}\right)\right)=U\left(x_{k}, u\left(x_{k}\right)\right)+\gamma J\left(x_{k+1}\right)
$$

where $\gamma \in(0,1)$ is discount factor and $U\left(x_{t}, u\left(x_{t}\right)\right) \geq 0$ is the utility function. According to the optimal principle of Bellman, the optimal performance index function $J^{*}\left(x_{k}\right)$ satisfies

$$
J^{*}\left(x_{k}\right)=\min _{u\left(x_{k}\right)}\left\{U\left(x_{k}, u\left(x_{k}\right)\right)+\gamma J^{*}\left(x_{k+1}\right)\right\},
$$

and $u^{*}\left(x_{k}\right)$ satisfies

$$
u^{*}\left(x_{k}\right)=\underset{u\left(x_{k}\right)}{\arg \min }\left\{U\left(x_{k}, u\left(x_{k}\right)\right)+\gamma J^{*}\left(x_{k+1}\right)\right\} .
$$

It is hard to solve (3) and (4) directly. ADP is an iterative algorithm. The algorithm is

$$
\begin{aligned}
& u_{i}\left(x_{k}\right)=\underset{u\left(x_{k}\right)}{\arg \min }\left\{U\left(x_{k}, u\left(x_{k}\right)\right)+\gamma J_{i}\left(x_{k+1}\right)\right\} \\
& J_{i+1}\left(x_{k}\right)=\min _{u\left(x_{k}\right)}\left\{U\left(x_{k}, u\left(x_{k}\right)\right)+\gamma J_{i}\left(x_{k+1}\right)\right\}
\end{aligned}
$$

where $u_{i}\left(x_{k}\right)$ is iterative control law, $J_{i}\left(x_{k}\right)$ is iterative performance index function, and $i=0,1,2, \cdots$ is iterative step. It had been proven in [15] that $J_{i}\left(x_{k}\right)$ is monotone nondecreasing and the algorithm will converge to the optimal while $J_{0}(x)$ is initiated as zero.

\section{Problem Formulation}

The stochastic optimal system of home energy in this paper is shown in Fig.1. There are one battery, uncontrollable appliances, and a optimal controller in the system. The controller controls the power of battery through an inverter and optimizes the energy of household.

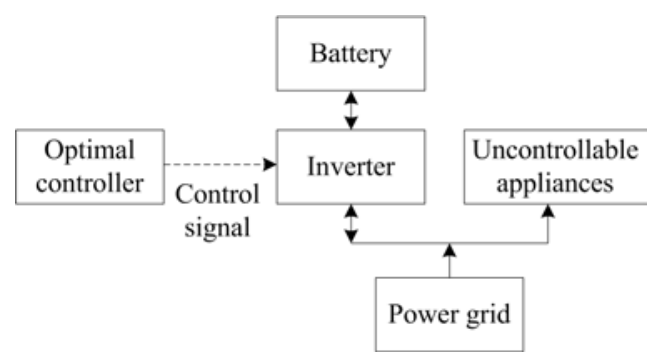

Fig.1. Stochastic optimal system of home energy

Let the time interval $\Delta T$ be one hour. There are 24 time steps in one day. Let $x_{k}$ be the energy of battery in time step $k, x^{\max }$ be the maximum energy of battery and $x^{\min }$ be the minimum one, $u_{k}$ be the power of battery in $k$ (positive while charging and negative while discharging), $\chi\left(u_{k}\right.$ ) be the efficiency of battery. The model of battery can be expressed as

$$
x_{k+1}=x_{k}+\chi\left(u_{k}\right) u_{k} \Delta T \text {. }
$$

Let $h_{k}$ be the total power consumption of uncontrollable appliance in time step $k, P_{k}(h)$ be the PDF of the total power consumption of uncontrollable appliance in $k, \beta_{k}$ be the dynamic price, $m_{1}$ and $m_{2}$ be the weights. The performance index function is

$$
J\left(x_{k}\right)=E_{u}\left\{\sum_{t=k}^{\infty} r^{t-k}\left[\beta_{t}^{2}\left(u_{t}+h_{t}\right)^{2}+m_{1}\left(x_{t}-\bar{x}\right)^{2}+m_{2} u_{t}^{2}\right]\right\}
$$

where $r \in(0,1)$ is discount factor and $\bar{x}=0.5\left(x^{\max }+x^{\min }\right)$. The first part of performance index function is power rate optimization. He we assume that the power is not allowed to be feedback to the power grid. The second part is the constraint to the energy of battery. Over charging or 
discharging is avoid by the constraint. The third part avoids the power of battery exceed its limit. As the probability of each time step is independent of each other, hence we have

$$
J\left(x_{k}\right)=\sum_{t=k}^{\infty} r^{t-k} \hat{U}\left(x_{t}, u_{t}, P_{t}, \beta_{t}\right)=\sum_{t=k}^{\infty} r^{t-k}\left\{\beta_{t}^{2} \sum_{h_{t}} P_{t}\left(h_{t}\right)\left(u_{t}+h_{t}\right)^{2}+m_{1}\left(x_{t}-\bar{x}\right)^{2}+m_{2} u_{t}^{2}\right\}
$$

$P_{k}$ and $\beta_{k}$ are not the input variables of $\hat{U}(\cdot)$. Let

$$
\hat{U}_{t}\left(x_{t}, u_{t}\right)=\hat{U}\left(x_{t}, u_{t}, P_{t}, \beta_{t}\right) \text {. }
$$

Then (9) can be written as

$$
J\left(x_{k}\right)=\sum_{t=k}^{\infty} r^{t-k} \hat{U}_{t}\left(x_{t}, u_{t}\right) .
$$

$\hat{U}_{t}(\cdot)$ is time-varying, but $U(\cdot)$ in (2) is time-invariant. ADP can not be applied directly to the problem.

\section{Elimination of Time-varying Parameter}

Based on the periodic feature, we assume $P_{k}(h)$ and $\beta_{k}$ are periodic functions of time, and their periods are all equal to one day. That is

$$
\beta_{j}=\beta_{j+24}, \quad P_{j}=P_{j+24}, j=k, k+1, \cdots \text {. }
$$

Define $v_{k}=\left\{u_{k}, u_{k+1}, \cdots, u_{k+23}\right\}$ and

$$
V_{k}\left(x_{k}, v_{k}\right)=\sum_{t=k}^{k+23} r^{t-k} \hat{U}\left(x_{t}, u_{t}, P_{t}, \beta_{t}\right) \text {. }
$$

According to (12), we have

$$
V_{k+24 L}(\cdot)=r^{24 L} V_{k}(\cdot), \quad L=0,1, \cdots \text {. }
$$

According to (13), (9) can be written as

$$
J\left(x_{k}\right)=V_{k}\left(x_{k}, v_{k}\right)+V_{k+24}\left(x_{k+24}, v_{k+24}\right)+V_{k+48}\left(x_{k+48}, v_{k+48}\right)+\cdots
$$

and

$$
J\left(x_{k}\right)=\sum_{L=0}^{\infty} r^{24 L} V_{k}\left(x_{k+24 L}, v_{k+24 L}\right)=V_{k}\left(x_{k}, v_{k}\right)+r^{24} J\left(x_{k+24}\right) .
$$

$V_{k}(\cdot)$ is time-invariant while $L$ changes. The influence of the time-varying parameters is eliminated. According to (7), the state transition equation for (16) is

$$
x_{k+24}=x_{k}+\Delta T\left(\chi\left(u_{k}\right) u_{k}+\cdots+\chi\left(u_{k+23}\right) u_{k+23}\right)=g\left(x_{k}, v_{k}\right) \text {. }
$$

Based on (12), the problem is transformed into (16) and (17), and ADP can be applied to solve it.

\section{A New Algorithm for The Problem}

Initialize $i=0$ and $J_{i}(x)=0$. According to ADP algorithm (5) and (6), the problem described by (16) and (17) is solved as

$$
\begin{aligned}
& v_{i}\left(x_{k}\right)=\underset{v_{k}}{\arg \min }\left\{V_{k}\left(x_{k}, v_{k}\right)+r^{24} J_{i}\left(x_{k+24}\right)\right\} \\
& J_{i+1}\left(x_{k}\right)=\min _{v_{k}}\left\{V_{k}\left(x_{k}, v_{k}\right)+r^{24} J_{i}\left(x_{k+24}\right)\right\} .
\end{aligned}
$$

Let $i \leftarrow i+1$, repeat (18) and (19) until $\left\|J_{j+1}\left(x_{k}\right)-J_{j}\left(x_{k}\right)\right\|<\varepsilon$ where $\varepsilon>0$ is a given precision. The ADP iteration is completed.

The computing burden of (18) is great as $\left\{u_{k}, u_{k+1}, \cdots, u_{k+23}\right\}$ must be computed simultaneously. (19) can be written as 


$$
J_{i+1}\left(x_{k}\right)=\min _{u_{k}, \cdots, u_{k+23}}\left\{\sum_{t=k}^{k+23} r^{t-k} \hat{U}\left(x_{t}, u_{t}, P_{t}, \beta_{t}\right)+r^{24} J_{i}\left(x_{k+24}\right)\right\} \text {. }
$$

This is a finite-horizon optimization problem that can be solve by DP. Here we propose a new algorithm. Let $t=k+23$ and $\hat{J}_{t+1}\left(x_{t+1}\right)=J_{i}\left(x_{k+24}\right)$, then compute

$$
\begin{aligned}
& u_{t}=\underset{u_{t}}{\arg \min }\left\{\hat{U}\left(x_{t}, u_{t}, P_{t}, \beta_{t}\right)+r \hat{J}_{t+1}\left(x_{t+1}\right)\right\} \\
& \hat{J}_{t}\left(x_{t}\right)=\min _{u_{t}}\left\{\hat{U}\left(x_{t}, u_{t}, P_{t}, \beta_{t}\right)+r \hat{J}_{t+1}\left(x_{t+1}\right)\right\} .
\end{aligned}
$$

Let $t \leftarrow t-1$, repeat (21) and (22) until $\hat{J}_{k}\left(x_{k}\right)$ is calculated. Then we have the solutions of (18) and (19) as

$$
\begin{aligned}
& v_{i}\left(x_{k}\right)=\left\{u_{k}, u_{k+1}, \cdots, u_{k+23}\right\} \\
& J_{i+1}\left(x_{k}\right)=\hat{J}_{k}\left(x_{k}\right)
\end{aligned} .
$$

\section{Numerical Results}

In this simulation, the initial time step is zero. That is $k=0$. The capacity of battery is $20 \mathrm{kWh}$. The initial energy of battery is $x_{0}=15 \mathrm{kWh} . \chi\left(u_{k}\right)=1, m_{1}=m_{2}=0.0005, r=0.95$. (12) is the key of the proposed algorithm. But in practice, the time-varying parameters will not keep periodic feature strictly. The average dynamic price of history is shown in Fig. 2 (a), and the dynamic price of the next 5 days is shown is Fig. 2 (b). There are 8 types of uncontrollable appliance in household. Their average switch-on probabilities are shown in Fig. 3 (a) (h). Their switch-on probabilities of the next 5 days are shown in Fig.3 (i) (p). This time-varying parameters in the future are similar to the average ones. We assume there is only one device of each uncontrollable appliance, and their power consumptions are unchanged while working, as shown in Table I.

Firstly the time horizon is set as 5 days.. It is assumed that the time-varying parameters of the next 5 days are known. We optimize the 5-day optimization problem by DP. The optimization results are the standard for the following comparisons. Notice that the time horizon of the standard should be infinite, but the simulation limits the setting.

As discussed in introduction, the infinite horizon problem is usually simplified into one-day horizon problem. Here we simplify the 5-day optimization problem into 5 one-day optimization problems, which called the simplified new problems based on DP (SNP-DP). We also apply the proposed algorithm to the infinite horizon problem. Two cases are considered in the following comparisons.

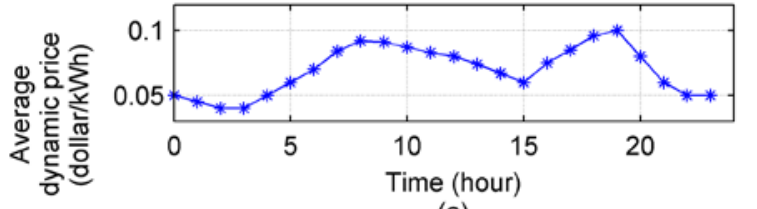

(a)

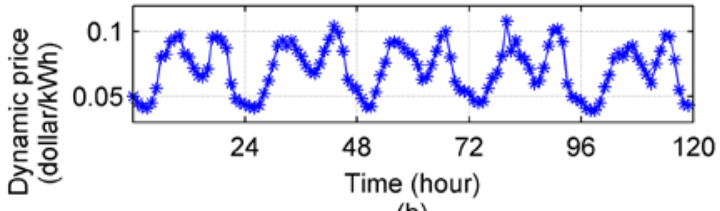

(b)

Fig.2. Dynamic price

Table I Power consumptions of uncontrollable appliance

\begin{tabular}{cc}
\hline Uncontrollable appliance & Power $(\mathrm{kW})$ \\
\hline Air conditioner & 3 \\
Computer & 0.29 \\
Washing machine & 2.5 \\
Cooking appliances & 1 \\
Fridge & 0.08 \\
Lighting & 0.3 \\
Electric shower & 3.5 \\
TV set & 0.25 \\
\hline
\end{tabular}



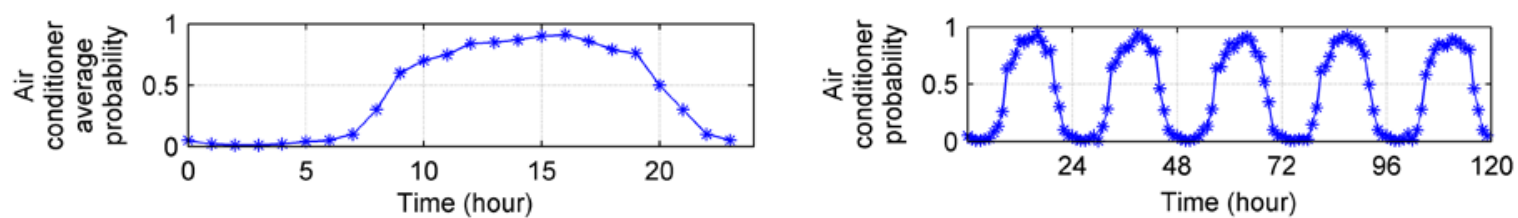

(a)
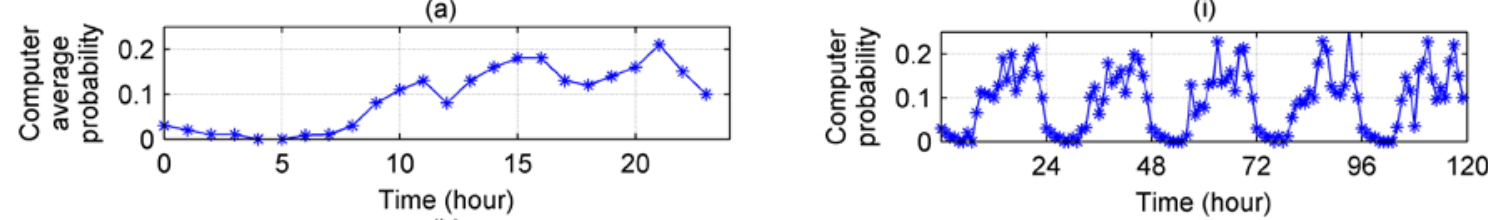

(b)

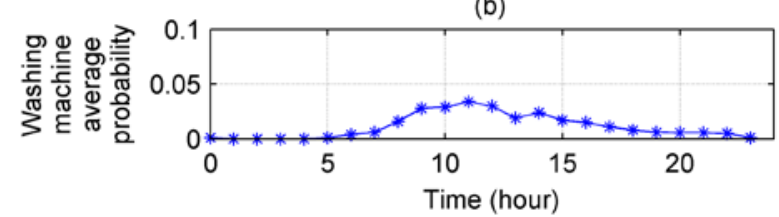

(c)

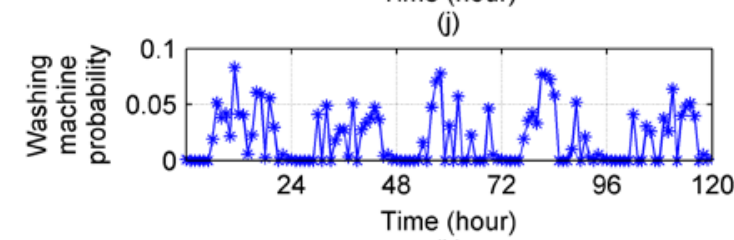

(k)
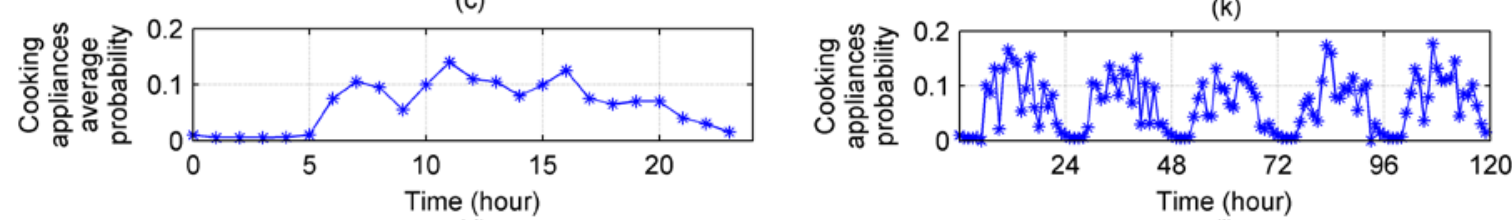

(I)
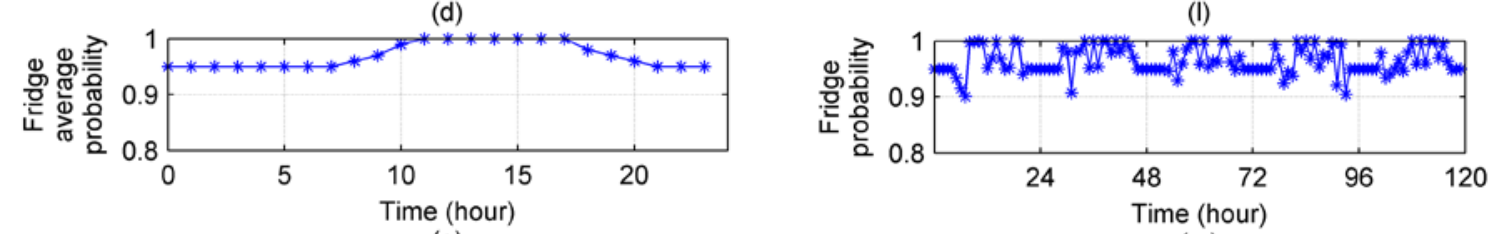

(e)

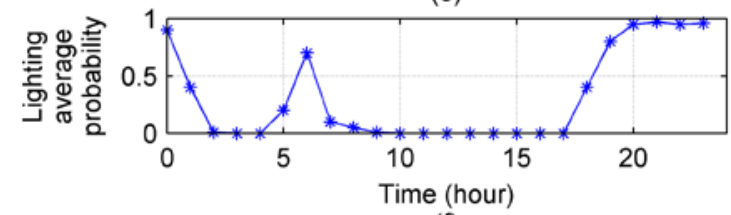

(f)

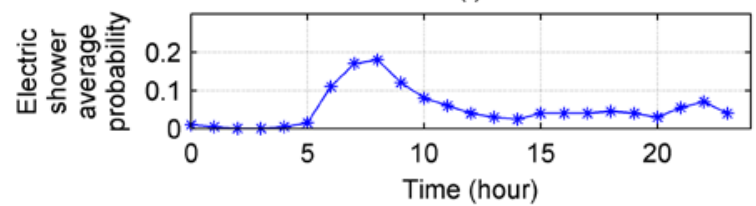

(g)

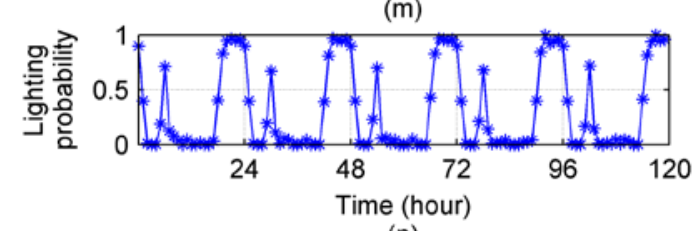

(n)

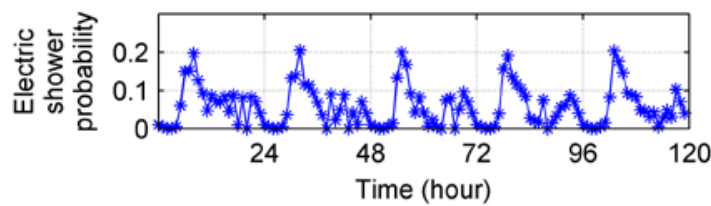

(o)

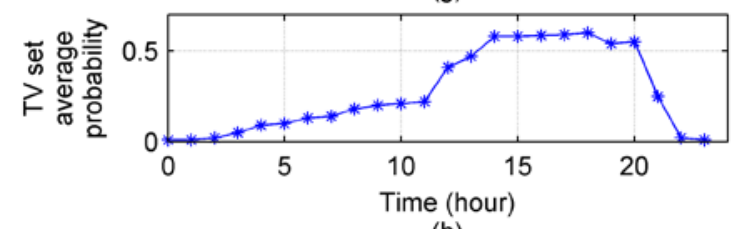

(h)

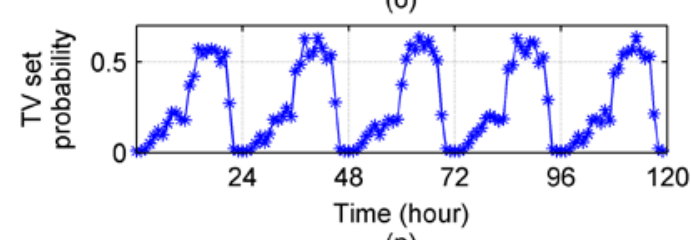

(p)

Fig.3. Switch-on probabilities of uncontrollable appliance

Case 1: the time-varying parameters in the future are unknown. They can not be predicted. We assume the time-varying parameters in the future are the same as the average ones of history. The results are shown in Fig. 4. The results of the proposed algorithm are similar to the ones of the standard. But the results of SNP-DP are not similar to the ones of the standard around 00:00 everyday. The reason is the cost after the end of time horizon is not considered in SNP-DP.

Case 2: the time-varying parameters of the next day are predicted precisely. We assume the time-varying parameters in the future are the same as the ones of the next day. The results are shown in Fig. 5. As the time-varying parameters in the future are known, the results of the proposed algorithm, the standard, and SNP-DP are almost the same. But as discussed in case 1, the results of SNP-DP are not similar to the ones of the standard in every midnight. 


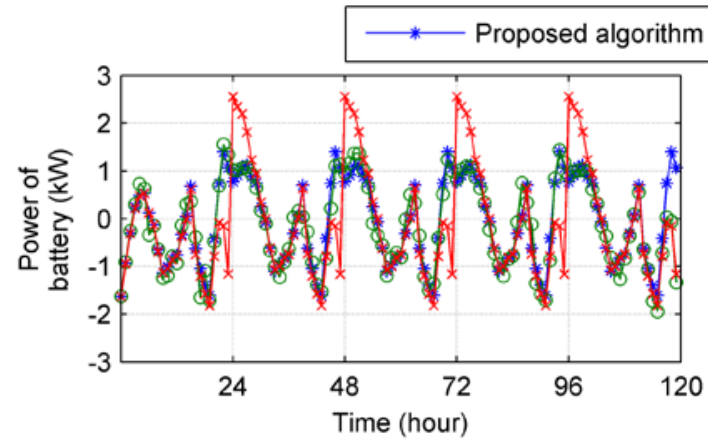

(a)

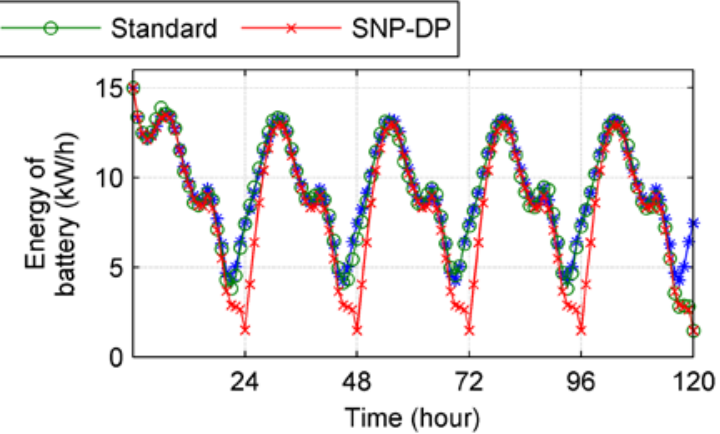

(b)

Fig.4. Optimal results without prediction

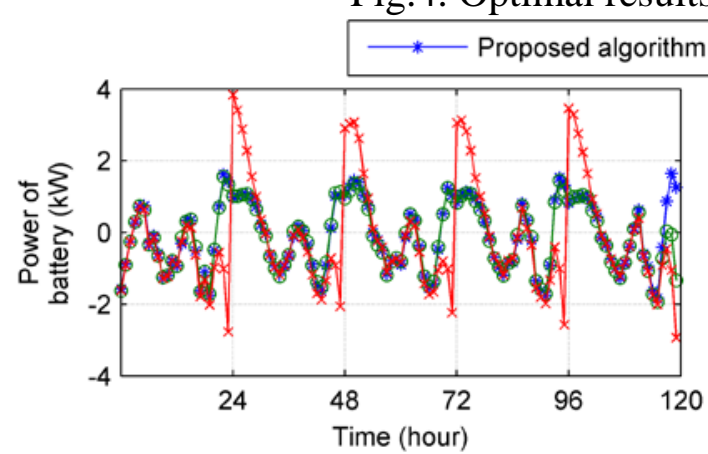

(a)

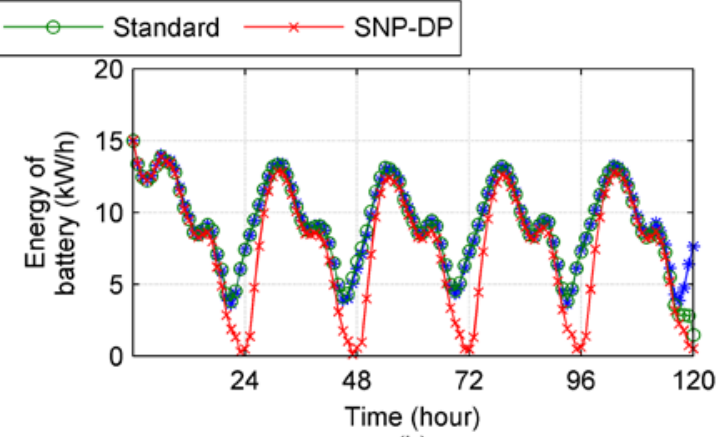

(b)

Fig.5. Optimal results with prediction

\section{Conclusion}

In this paper, a new ADP algorithm is proposed to solve the infinite horizon HEO problem. Simulation results show that the proposed algorithm has great performance. On the other hand, there are batteries within electric vehicles. electric vehicle is a good storage equipment in HEO. The ADP application for HEO with electric vehicles is the future study of the paper.

\section{Acknowledgement}

In this paper, the research was sponsored by the National Natural Science Foundation of China (Project No. U1501251 and 51307025) and Ph.D. Programs Foundation of the Ministry of Education of China (Project No. 20124420130001).

\section{References}

[1] Z. Yu, L. Jia, M. C. Murphy-hoye, et al. Modeling and stochastic control for home energy management [J]. IEEE Transactions on Smart Grid, 2013, 4(4): 2244-2255.

[2] F. Y. Xu, T. Zhang, L. L. Lai, et al. Shifting boundary for price-based residential demand response and applications [J]. Applied Energy, 2015, 146: 353-370.

[3] M. H. K. Tushar, C. Assi, M. Maier, et al. Smart microgrids: optimal joint scheduling for electric vehicles and home appliances [J]. IEEE Transactions on Smart Grid, 2014, 5(1): 239-250.

[4] Lei Zhou, Yang Li. Modeling and optimal dispatch for residential load based on home energy management system under time-of-use pricing [J]. Power System Technology, 2015, 39(2): 367-374.

[5] Y. Wang, X. Lin, M. Pedram. Adaptive control for energy storage systems in households with photovoltaic modules [J]. IEEE Transactions on Smart Grid, 2014, 5(2): 992-1001.

[6] R. Deng, Z. Yang, J. Chen, et al. Residential energy consumption scheduling: a 
coupled-constraint game approach [J]. IEEE Transactions on Smart Grid, 2014, 5(3): 1340-1350.

[7] Ciwei Gao, Qianyu Li, Yang Li. Bi-level optimal dispatch and control strategy for air-conditioning load based on direct load control [J]. Proceedings of the CSEE, 2014, 34(10): 1546-1555.

[8] R. E. Bellman. Dynamic programming [M]. Princeton, NJ, USA: Princeton University Press, 1957.

[9] P. J. Werbos. Neural networks for control and system identification [J]. Proceedings of the 28th IEEE Conference on Decision and Control, 1989, 1: 260-265.

[10] J. J. Murray, C. J. Cox, G. G. Lendaris, et al. Adaptive dynamic programming [J]. IEEE Transactions on Systems, Man, and Cybernetics, Part C, 2002, 32(2): 140-153.

[11] P. J. Werbos. Computational intelligence for the smart grid - history, challenges, and opportunities [J]. IEEE Computational Intelligence Magazine, 2011, 69(3): 14-21.

[12] G. K. Venayagamoorthy. Dynamic, stochastic, computational, and scalable technologies for smart grids [J]. IEEE Computational Intelligence Magazine, 2011, 6(3): 22-35.

[13] R. N. Anderson, A. Boulanger, W. B. Powell, et al. Adaptive stochastic control for the smart grid [J]. Proceedings of the IEEE, 2011, 99(6): 1098-1115.

[14] Derong Liu, Qinglai Wei. Policy iteration adaptive dynamic programming algorithm for discrete-time nonlinear systems [J]. IEEE Transactions on Neural Networks and Learning Systems, 2014, 25(3): 621-634.

[15] Ding Wang, Derong Liu, Qinglai Wei, et al. Optimal control of unknown nonaffine nonlinear discrete-time systems based on adaptive dynamic programming [J]. Automatica, 2012, 48(8): 1825-1832. 